\title{
COMPARISON OF PATHOLOGICAL CHANGES IN THE STUDY OF DOGS AFFECTED BY PARVOVIRAL ENTERITIS AND INTESTINAL YERSINIOSIS
}

\author{
Hryhorii Zon \\ Department of Virology, Pathological anatomy and Poultry Diseases ${ }^{l}$ \\ zon_g@ukr.net \\ Lyudmyla Ivanovska \\ Department of Virology, Pathological anatomy and Poultry Diseases ${ }^{l}$ \\ lusj0951@gmail.com \\ Illia Zon \\ Department of Department of Veterinary Examination, Microbiology, \\ Zoohygiene and Safety and Quality of Livestock Products ${ }^{1}$ \\ zonillya@hotmail.com \\ Tion Matthew Terzungwe \\ Department of Virology, Pathological anatomy and Poultry Diseases ${ }^{I}$ \\ tions_doc@yahoo.co.uk \\ ${ }^{1}$ Sumy National Agrarian University \\ 160 Herasyma Kondratieva str., Sumy, Ukraine, 40021
}

\footnotetext{
Abstract

Aim of the study: to examine the pathological changes in dogs that died due to confirmed intestinal yersiniosis and parvoviral enteritis and establish trends for characteristic organ lesions for both diseases in comparison, then evaluate our findings with the existing published material of sufficient evidence quality regarding differential postmortem diagnosis of spontaneous intestinal yersiniosis and parvoviral enteritis in dogs.

Materials and methods: the study examined the cases of $25 \mathrm{dogs}$ from 2 month to $1.3 \mathrm{y}$. o. of various breed, gender that died due to either confirmed Canine parvoviral enteritis (CPV) infection or intestinal yersiniosis and subsequently divided into two groups based on their diagnosis.

The definitive diagnosis has been confirmed by performing rapid ELISA diagnostics (SensPERT ${ }^{\circledR}$, VetAll Laboratories, Kyunggi-Do, Korea) for CPV and serological tests for Y. enterocolitica sera AT were performed using yersiniosis antigens from the «Component set for serological diagnosis of animal yersiniosis» (Kharkiv, NSC IEKVM, TS 46.15.091-95) in accordance with the «Interim guidelines for the use of a set of components for serological diagnostics». A dilution of 1:200 has been considered as the diagnostic titer.

After the definitive diagnosis had been confirmed, the animals were divided into two groups, depending on diagnosis: CPV $(n=14)$ or IY $(n=11)$. The autopsies of twenty randomly (10 from each group) selected dog corpses have been conducted utilizing standard methodology.

Results: according to the results of autopsy of dogs afflicted by CPV, the main pathological changes were found in the small intestine - catarrhal-desquamative inflammation (in $100 \%$ of cases), serous-hemorrhagic mesenteric lymphadenitis (90\%) large intestine $(70 \%)$ in the stomach (60\%), in the liver (50\%), in other organs - less than 40 percent of cases and most notably caused lung damage (edema and local atelectasis) in $90 \%$ of the animals in the study, which was not the case for intestinal yersiniosis with only $20 \%$ incidence of lung damage.

Conclusions: Spontaneous intestinal yersiniosis in dogs was pathologically manifested by pronounced catarrhal-desquamative processes mainly in the stomach and small intestine (70,100 and $80 \%$ of cases, respectively), inflammation of the mesenteric lymph nodes $(90 \%)$ and large intestine $(80 \%)$, dystrophy and congestive processes in the liver (80 \%). Low incidence and type of lung damage (congestive hyperemia in $20 \%$ of reviewed cases compared to $90 \%$ of local atelectasis add edema in CPV group) was noteworthy. Cases of 25 animals that died due to either confirmed CPV or Y. enterocolitica infection were analyzed, and 20 animal corpses were autopsied during study. Dogs that died from intestinal yersiniosis had significantly higher frequency of pathological findings in kidney (200\%) and liver (60\%) in particular. Changes in stomach and large intestine were also more frequent. At the same time, we observed a lower frequency of pathological changes in spleen (33\% lower), heart (25\% lower) and the lowest
} 
frequency was in lungs (77\% lower) presented by edema and local atelectasis in animals afflicted by yersiniosis compared to CPV. Incidence and manifestation of pathological findings was mesenteric lymph nodes and abdominal cavity were similar, and could not be considered during posthumous diagnostics.

Keywords: intestinal yersiniosis, parvoviral enteritis, dogs.

DOI: $10.21303 / 2504-5679.2021 .001690$

\section{Introduction}

Corpses of the dogs, which died due to confirmed intestinal yersiniosis or canine parvoviral enteritis, were autopsied to determine comparative pathological changes in the internal organs.

Canine parvoviral enteritis is an acute, contagious disease that can be easily transmitted among sensitive dogs. Parvoviral enteritis can be spread by direct contact with patients' feces, oronasal route or by indirect contact with contaminated media (contaminated dog food, food bowls and other utensils, bedding, shoes, clothing, human hands). CPV is usually diagnosed in places of concentration of dogs, especially at exhibitions, tests of service dogs, in nurseries, animal shelters, parks and playgrounds. Some flies, including domestic flies (Muscidae, Sarcophagidae, Calliphoridae), are involved in virus transmission. CPV affects the gastrointestinal tract, heart, liver, kidneys and other organs. It is clinically accompanied by vomiting, melena, dehydration, ataxia, and complications of the underlying disease and other symptoms.

Intestinal yersiniosis transmission (Yersinia enterocolitica) also occurs predominately through fecal-oral route. However, how often this happens, what conditions contribute to this process is almost unknown. Various disorders of the gastrointestinal tract lead to dehydration, severe depression, melena and in some cases abortions in pregnant bitches. The autopsy reveals mainly pathology of the gastrointestinal tract, liver. It causes changes in other organs in the associated course with other diseases. Difficulties in recognizing the disease are mainly due to the fact that the disease is often misdiagnosed or it is asymptomatic for some time.

Pathological changes in dogs afflicted with CPV have been described by researchers from different countries. Thus, in Australia, after autopsy of dogs with enteritis, $30 \%$ of death cases were attributed to canine parvovirus, $2.6 \%$ of cases to distemper, and in $2 \%$ of cases - to coronavirus [1]. Postmortem changes caused by the intestinal form of the disease in dogs that have died from CPV may include dehydration, empty bowel, or reddish fluid contents [2]. Decaro N. and Buonavoglia C. reported inflammation of the small intestine, enlargement of the mesenteric lymph nodes. Multifocal hemorrhages and exudates, congestive venous hyperemia and edema were recorded in the lungs and trachea as well as the atrophy of the thymus [3]. Purulent exudates were also observed along the ventral part of the skull, the optic nerve chiasm, the medulla oblongata, and in the ventricles of the brain [4]. Microscopic lesions of the gastrointestinal tract include dystrophy, necrosis and atrophy of the villi, thickening of the duodenal mucosa, roughness and curvature of the villi and changes in their morphology, neutrophilic and lymphocytic infiltration of the intestinal mucosa, submucosal layers. In acute cases of CPV-2 pulmonary tissue may be characterized by diffuse alveolar edema, neutrophilia, and numerous accumulations of opportunistic bacteria [5]. Large necrotic lesions and mononuclear perivascular cuffs in white and gray matter are found in the brain [6].

The necropsy revealed acute myogenic dilation of the heart, pale and motley coloring of the myocardium on an incision surface, loss of myofibrils, lysis of myocytes and liquid accumulation in the pericardium or thoracic cavity. Older dogs who have survived a neonatal infection usually develop scars with myocardial thinning [3, 7]. Histologically, puppy myocarditis is characterized by the presence of cytoplasmic intranuclear inclusions in the nuclei of myocardial cells, fibrous, necrotic and alterative myocarditis with high lymphocyte infiltration. Some researchers believe that myocarditis of parvoviral etiology can be clearly differentiated from other deadly diseases that affect puppies according to the results of pathomorphological examination [8]. Pathomorphological signs of the myocardial form of parvovirus infection in dogs were found in puppies aged 1 to 4 weeks who died suddenly without clinical signs and biochemical changes in the blood characteristic of myocarditis [9]. Histological examination revealed typical morphological changes: lymphoid (non-purulent) myocarditis; eosinophilic inclusion bodies in the nuclei of cardiomyocytes; edema of the stroma of 
the heart; granular dystrophy and necrosis of cardiomyocytes with destruction of myocardial muscle fibers. Also non-specific permanent morphological signs arising from circulatory disorders and heart failure were encountered like venous hyperemia of the liver, kidneys and pulmonary edema [9, 10]. At the same time, mutations in viruses that cause CPV in dogs in recent times, somewhat change both the mechanisms of pathogenesis and pathological manifestations of the disease [4, 11].

In canine intestinal yersiniosis, along with the asymptomatic course of the disease and fecal shedding of the pathogen, clinically pronounced forms of the disease are determined, accompanied by diarrhea, joint damage and cases of a generalized condition, leading to death [12-14]. Most reports indicate the dominance, prevalence and growth of pathogenicity of Y. enterocolitica, among other biovars of yersinia. Therefore, when describing the pathological picture of yersiniosis, the greatest attention was paid to intestinal yersiniosis. In addition, this is due to the important epidemiological significance of the disease. In published materials for intestinal yersiniosis described at autopsy catarrhal-hemorrhagic gastroenterocolitis, serous peritonitis, synovium, female genital lesions $[15,16]$, rarely other pathologies [17], especially with complications of the underlying disease.

Express-diagnosis of these diseases is not always effective, due to the presence of a certain amount of antibodies and other reasons [18]. Thus, the information provided indicates the need to have criteria for pathological assessment of two similar clinical manifestations of diseases of different etiologies.

The aim of the study was to identify the comparative incidence of pathological signs caused by CPV and intestinal yersiniosis that can enable practitioners to conduct a differential postmortem diagnosis.

\section{Materials and methods of research}

The study analyzed cases of 25 dogs of various breeds, gender and weight, aged from $2 \mathrm{~m}$. to 1.3 y. o., which were admitted to veterinary clinics «Hels» and «Vetservis» in Sumy, Ukraine throughout 2019-2020. The dogs presented with melena, severe dehydration, vomiting, various levels of ataxia and perished during the course of the disease. The work was performed in accordance with the research programs of Sumy National Agrarian University: «To develop an epizootic welfare control system for infectious animal diseases based on monitoring, diagnosis, forecasting and safety assessment of livestock and poultry products in North-Eastern Ukraine» (No. DR 0114U000261). The work was carried out for the period from 2019 to 2020 at the Department of Virology, Pathological Anatomy and Poultry Diseases of Sumy National Agrarian University branch, located at a small animal clinic «Vetservis» Sumy, Ukraine.

The definitive diagnosis has been confirmed by performing rapid ELISA diagnostics (SensPERT $^{\circledR}$, VetAll Laboratories, Kyunggi-Do, Korea) for CPV and serological tests for Y. enterocolitica sera AT were performed using yersiniosis antigens from the «Component set for serological diagnosis of animal yersiniosis» (Kharkiv, NSC IEKVM, TS 46.15.091-95) in accordance with the «Interim guidelines for the use of a set of components for serological diagnostics» approved by HOVM with the State Inspectorate on November 16, 1995 according to No. 15-14/52). A dilution of 1:200 has been considered as the diagnostic titer.

After the definitive diagnosis had been confirmed, the animals were divided into two groups, depending on diagnosis: CPV $(n=14)$ or IY $(n=11)$. The autopsies of twenty randomly (10 from each group) selected dog corpses have been conducted utilizing standard methodology [19] at «Vetservice» veterinary clinic in Sumy. During the autopsies, the following criteria were taken into account: the location and type of lesions (Tables 1, 2), frequency of occurrence of different lesions in each group (Tables 1, 2).

Pathoanatomical research was conducted on diseased animals. The animal corpses were stored in laboratory freezers at $-5{ }^{\circ} \mathrm{C}$. After the autopsy all the specimens were destroyed in accordance with Cabinet Of Ministers Of Ukraine decree No. 896 from 4.11.2015 by means of Beccari pit. The autopsy facility and instruments were decontaminated using $2 \%$ solution of «Chloramin-B» (Schulke \& Mayr GmbH). The research adheres to ARRIVE guidelines and Directive 2010/63/EU of the European Parliament and of the Council of 22 September 2010 on the protection of animals used for scientific purposes. 
We used percentage calculation to compare the incidence of different pathoanatomical phenomena in both research groups, results were statistically analyzed using Mann-Whitney U criteria. The results were calculated using Microsoft Excel 2016.

\section{Results}

During the autopsy of dogs afflicted with CPV mild stage of rigor mortis was observed. Serous fluid or crusts of mucus or dried serous exudate were found around the nose, and around the mouth - varying from gray to pale pink. The anal area and hind limbs were contaminated with liquid bloody feces. Dissection of the corpses revealed effusion into the abdominal cavity. In the stomach, the mucous membrane was grayish-red with hemorrhages. Inflammation of the gastric mucosa was localized mainly on the folds (Fig. 1).

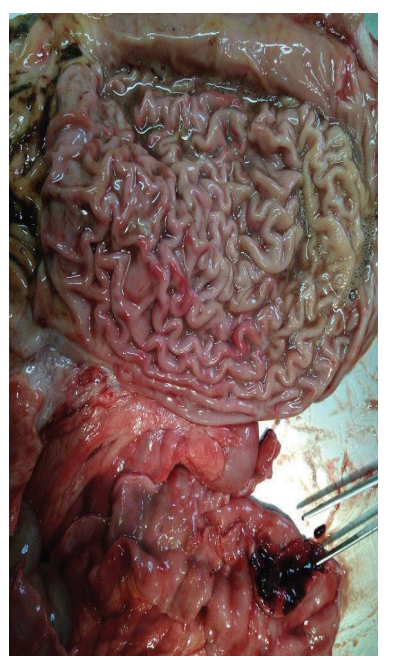

Fig. 1. Catarrhal - hemorrhagic inflammation of the gastric mucosa in a dog that died from Y. enterocolotica infection

The intestine, where the main pathological changes were localized, was almost void of content. The mucous membrane was in a state of acute catarrhal-hemorrhagic desquamative inflammation (Fig. 2, a, b), mainly in the small intestine. Also, some areas with erosive hemorrhagic lesions were spotted. In some cases, peeling of the mucous membrane was observed in areas of several centimeters.

The liver was enlarged, elastic and firm consistency, brown, with grey focal lesions on the surface of a parenchymal cut (Fig. 3).

Visible anatomical changes in the kidneys were almost not determined. There were hemorrhages in the spleen.

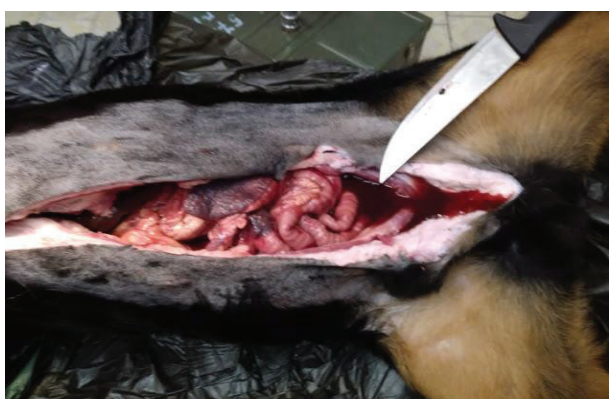

$a$

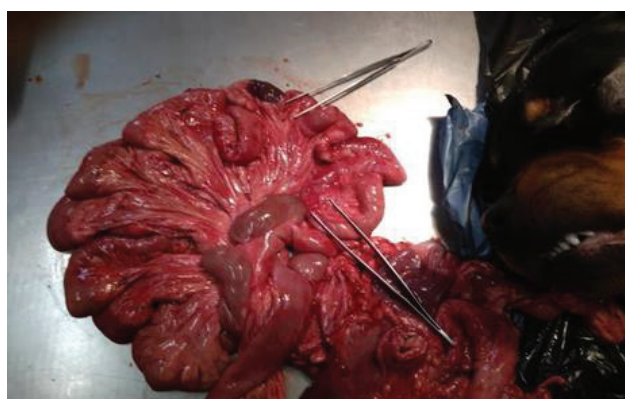

$b$

Fig. 2. Pathological signs of CPV: $a$ - inflammation of the intestine in an animal that died from CPV; $b$ - catarrhal inflammation of the intestinal mucosa and mesenteric lymphadenitis 


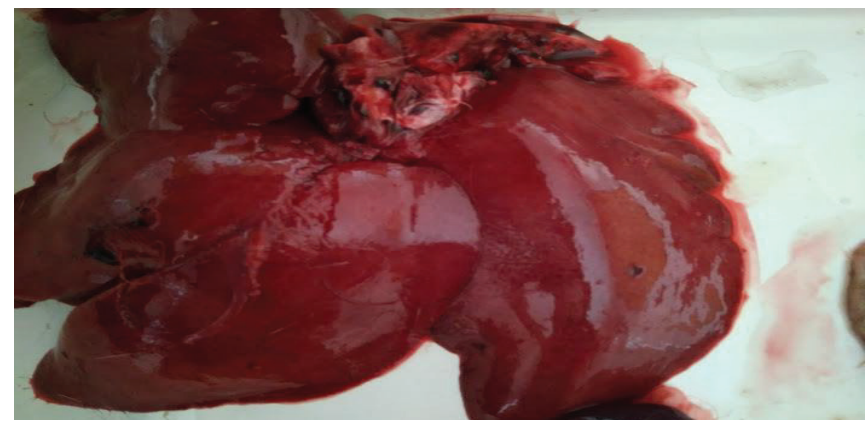

Fig. 3. Granular dystrophy of the dog's liver with CPV

Pleural hemorrhages, foci of atelectasis and pulmonary edema were detected. The lungs were mostly elastic and loose when pressed. The incision surfaces of both lungs were spotted with hemorrhages (Fig. 4).

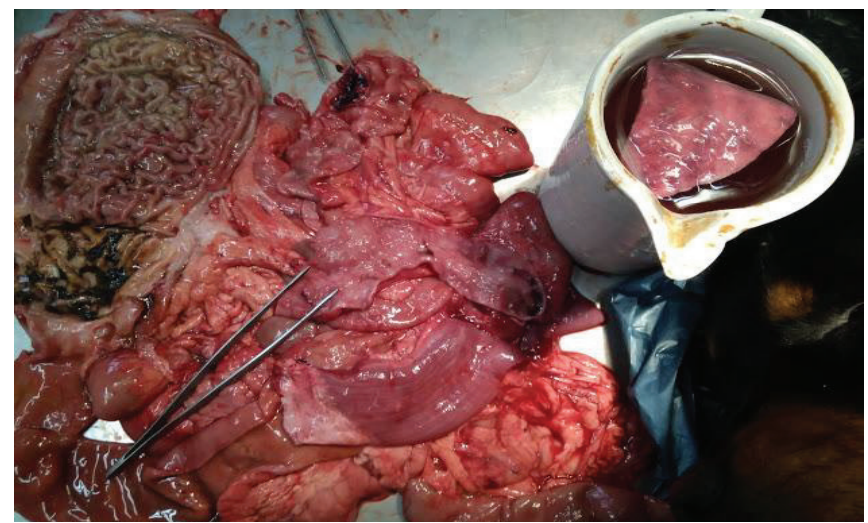

Fig. 4. Lesions in canine lungs with $\mathrm{CPV}$

The results of the generalization of the pathological and anatomical picture in the study of dogs that died from CPV are presented in Table 1.

\section{Table 1}

Frequency of pathological findings in the organs of dogs that died from CPV, $n=10, p>0.05$

\begin{tabular}{lccl}
\multicolumn{1}{c}{ Organs } & Number of cases & $\mathbf{\%}$ & \multicolumn{1}{c}{ Pathological changes } \\
\hline Stomach & 6 & 60.0 & Acute catarrhal inflammation \\
Small intestine & 10 & 100.0 & Acute catarrhal-hemorrhagic, desquamative inflammation \\
Large intestine & 7 & 80.0 & Catarrhal (rarely catarrhal-hemorrhagic) inflammation \\
Mesenteric lymph nodes & 9 & 90.0 & Serous, serous-hemorrhagic lymphadenitis \\
Spleen & 3 & 30.0 & Hemorrhages \\
Liver & 5 & 50.0 & Focal granular dystrophy \\
Lungs & 9 & 90.0 & Edema, atelectasis, redness \\
Heart & 4 & 40.0 & Acute enlargement, granular dystrophy \\
Kidney & 2 & 20.0 & Granular dystrophy \\
Chest cavity & 3 & 30.0 & Hemorrhages \\
Abdominal cavity & 4 & 40.0 & Hemorrhages
\end{tabular}


Analysis of pathological and anatomical findings shows that despite the age difference (from 2.5 months to 1.5 years) of animals the majority of subjects demonstrated significant damage of the abdominal organs. The severity of pathological changes depended primarily on the duration of the clinical period and the severity of the course.

In most cases, the heart muscle damage could not be detected during an autopsy, but this does not exclude changes in the organ at the tissue level. Hemorrhages were found in the thoracic and abdominal cavities in 20-30\% of cases. The variety of changes in the lungs (from hyperemia and edema to focal atelectasis), which was observed in $90 \%$ of cases, is noteworthy.

Pathological examination of dogs with spontaneously occurring yersiniosis revealed mainly catarrhal gastroenteritis, catarrhal-hemorrhagic gastroenteritis or enterocolitis, characterized by uneven hyperemia of the mucous membrane, loss of luster, swelling, loosening and excessive folding (Fig. 5, $\boldsymbol{a}, \boldsymbol{b} ; \mathbf{6}, \boldsymbol{a}, \boldsymbol{b}$ ) with excessive accumulation of turbid mucus in the form of strands or clots. The contents were mostly liquid, cloudy, laced with mucus and often contain blood impurities. The mesentery vessels were overflowing with blood; the lymph nodes were enlarged, with their dissection revealling signs of serous lymphadenitis and hyperemia (Fig. 7, $\boldsymbol{a}, \boldsymbol{b}$ ).

Examination of the liver revealed deep dystrophic processes with blunting of the edges of the organ, discoloration and increased tension of the capsule. The color of the parenchyma is heterogeneous varying from dark cherry to clay. Dissection reveals, smoothed parenchymal pattern and a scrape giving off moderate amount of content. The gall bladder was overflowing with bile in all cases.

The kidneys showed an insignificant increase in volume, mainly due to congestive hyperemia. Other changes were found less frequently (Table 2).

Upon receiving data from the autopsies and incidence percent calculation, a percent bias was determined (Table 3).

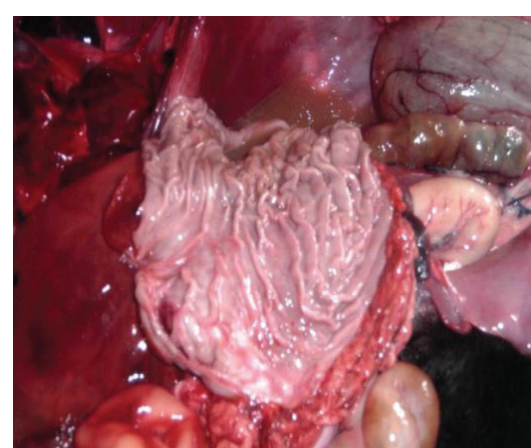

$a$

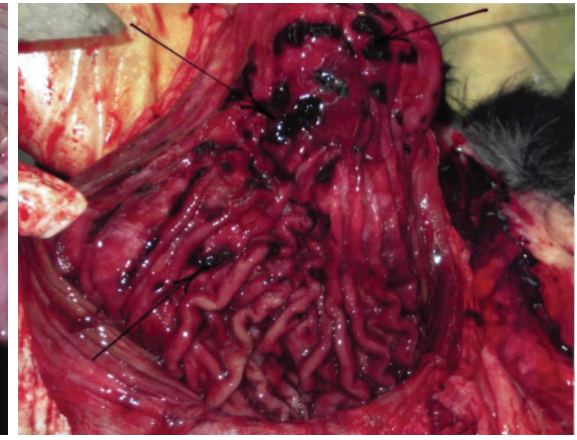

$b$

Fig. 5. Inflammatory processes in the stomach of dogs with intestinal yersiniosis: $a$-petechial effusions in the stomach mucosa; $b$ - catarrhal - hemorrhagic inflammation of the stomach mucosa

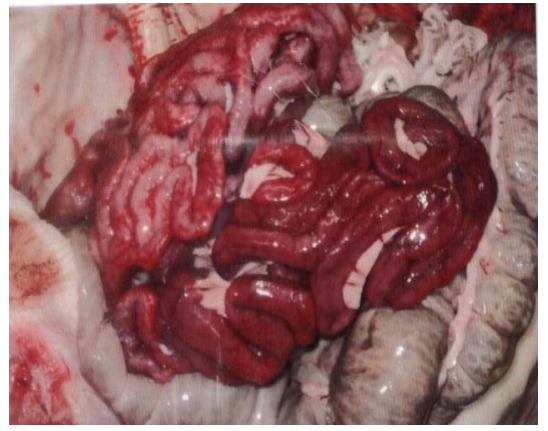

$a$

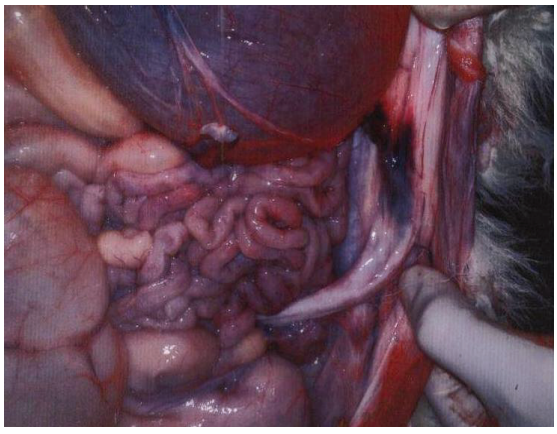

$b$

Fig. 6. Acute catarrhal enterocolitis in intestinal yersiniosis: $a$ - catarrhal enterocolitis; $b$ - ischemic lesions, localized catarrhal lesions 


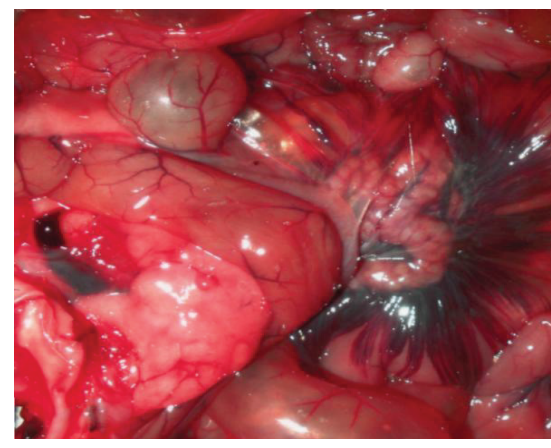

a

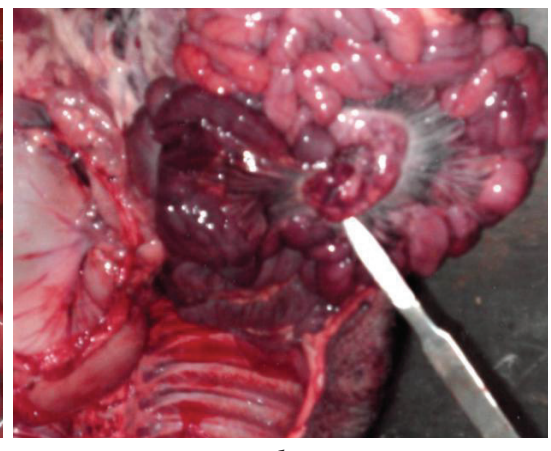

$b$

Fig. 7. Inflammation of the mesenteric lymph nodes: $a$-mesenteric lymphadenitis; $b$ - necrotic lesions in a mesenteric lymph node, fragment of an inflamed bowel

Table 2

Frequency of pathological findings in the organs of dogs that died from intestinal yersiniosis, $n=10, p>0.05$

\begin{tabular}{lccl}
\hline \multicolumn{1}{c}{ Organs } & Number of cases & $\%$ & \multicolumn{1}{c}{ Pathological changes } \\
\hline Stomach & 7 & 70 & Catarrhal, catarrhal-hemorrhagic gastritis \\
Small intestine & 10 & 100 & Catarrhal, catarrhal-hemorrhagic enteritis \\
Large intestine & 8 & 80 & Catarrhal, catarrhal-hemorrhagic colitis \\
Mesenteric lymph nodes & 9 & 90 & Serous lymphadenitis \\
Spleen & 2 & 20 & Congestive hyperemia \\
Liver & 8 & 80 & Congestive hyperemia, granular dystrophy \\
Lungs & 2 & 20 & Congestive hyperemia \\
Heart & 3 & 30 & Granular dystrophy \\
Kidney & 6 & 60 & Congestive hyperemia, granular dystrophy \\
Chest cavity & 1 & 10 & Fluid effusion \\
Abdominal cavity & 4 & 40 & Congestive hyperemia of the mesenteric vessels, fluid effusion
\end{tabular}

Table 3

Percent bias calculation for the incidence of pathological changes occurrence in different organ systems during intestinal yersiniosis vs CPV infection

\begin{tabular}{lccc}
\hline \multicolumn{1}{c}{ Organs } & CPV & Yersiniosis & Percent bias \\
\hline Stomach & 6 & 7 & 0.17 \\
Small intestine & 10 & 10 & 0.00 \\
Large intestine & 7 & 8 & 0.14 \\
Mesenteric lymph nodes & 9 & 9 & 0.00 \\
Spleen & 3 & 2 & -0.33 \\
Liver & 5 & 8 & 0.60 \\
Lungs & 9 & 2 & -0.78 \\
Heart & 4 & 3 & -0.25 \\
Kidney & 2 & 6 & 2.00 \\
Chest cavity & 3 & 1 & -0.67 \\
Abdominal cavity & 4 & 4 & 0.00
\end{tabular}




\section{Discussion}

The literature analysis revealed insufficient data regarding small animal yersiniosis, especially in the aspect of pathoanatomical diagnostics, which shows current lack of knowledge in this area possibly because of main research efforts being directed towards food safety and prevention of disease spread.

Comparative pathological studies have shown that it is extremely difficult to detect significant differences in the incidence of lesions of the gastrointestinal tract. Interestingly, for CPV, the phenomena of lung hyperemia, local atelectasis and edema were found in $90 \%$ of cases, against $20 \%$ of cases of congestive hyperemia in intestinal yersiniosis, which corresponds with available data from other sources $[6,20]$. At the same time examination of pleura and peritoneum in CPV revealed hemorrhages, which was not observed in intestinal yersiniosis.

Pathologically, both diseases show signs of granular dystrophy in the heart and kidneys further corroborating previous studies [4, 9]. Moreover, CPV manifests pronounced desquamative processes, often with detachment of the mucous membrane on the background of the inflammatory process [3], which was not observed in intestinal yersiniosis [10, 20]. In the case of bloody diarrhea, when the stools contain only small pieces of epithelium, the accurate clinical diagnosis is problematic [5].

Hemorrhages can be found in the body during CPV, but venous hyperemia was only detected in two cases of intestinal yersiniosis. Interestingly, for CPV, the phenomena of hyperemia, local atelectasis and edema were found in $90 \%$ of cases, against $20 \%$ of cases of congestive hyperemia in intestinal yersiniosis. Pathologically, both diseases show signs of granular dystrophy in the heart and kidneys.

Depending on the timing of the disease, the use of specific diagnostic tests may also be unsuccessful [2] and definitive tests for Y. enterocolitica require bacterial cultures or sera titer diagnostics, which can be time-consuming and ineffective as a rapid diagnostic test in-vivo [12].

Research limitations. The diagnostic potential of the described method is limited due to:

1. Small quantity of specimens, which is a result of seldomly conducted autopsies of deceased patients at small animal clinics due to absence of mandatory autopsy provisions in veterinary regulatory documents, inability to properly perform the autopsy or to adhere to proper pathological material disposal protocol and ethical concerns of the owners, unwilling to give their consent for an autopsy. Further studies will enable proper statistical analysis of incidence of different pathoanatomic findings.

2. Potential concurrent course of both diseases and/or presence of secondary or underlying pathological process/status severely limits the possibility of accurate pathoanatomical diagnosis.

Further studies. Further research will be directed towards the increase of the quantity of autopsies being performed on target population animals in order to perform more accurately evaluations of the incidence of certain pathological changes in various organs and systems which will result in more accurate postmortem diagnostics.

Potentially, concurrent course of CPV infection and intestinal yersiniosis can be studied to determine the impact of secondary bacterial infections on the outcome of CPV.

\section{Conclusions}

1. Cases of 25 animals that died due to either confirmed CPV or Y. enterocolitica infection were analyzed, and 20 animal corpses were autopsied during study.

2. Dogs that died from intestinal yersiniosis had significantly higher frequency of pathological findings in kidney (200\%) and liver (60\%) in particular. Changes in stomach and large intestine were also more frequent.

3. At the same time, we observed a lower frequency of pathological changes in spleen (33\% lower), heart ( $25 \%$ lower) and the lowest frequency was in lungs ( $77 \%$ lower) presented by edema and local atelectasis in animals afflicted by yersiniosis compared to CPV.

4. Incidence and manifestation of pathological findings was mesenteric lymph nodes and abdominal cavity were similar, and could not be considered during posthumous diagnostics. 


\section{Conflicts of interest}

The authors declare that they have no conflicts of interest.

\section{References}

[1] Kelman, M., Norris, J., Barrs, V., Ward, M. (2020). A history of canine parvovirus in Australia: what can we learn? Australian Veterinary Journal, 98 (10), 504-510. doi: http://doi.org/10.1111/avj.13002

[2] Goddard, A., Leisewitz, A. L. (2010). Canine Parvovirus. Veterinary Clinics of North America: Small Animal Practice, 40 (6), 1041-1053. doi: http://doi.org/10.1016/j.cvsm.2010.07.007

[3] Decaro, N., Buonavoglia, C. (2011). Canine Coronavirus: Not Only an Enteric Pathogen. Veterinary Clinics of North America: Small Animal Practice, 41 (6), 1121-1132. doi: http://doi.org/10.1016/j.cvsm.2011.07.005

[4] Agungpriyono, D. R., Uchida, K., Tabaru, H., Yamaguchi, R., Tateyama, S. (1999). Subacute Massive Necrotizing Myocarditis by Canine Parvovirus Type 2 Infection with Diffuse Leukoencephalomalacia in a Puppy. Veterinary Pathology, 36 (1), 77-80. doi: http://doi.org/10.1354/vp.36-1-77

[5] Nwoha, R. I. O. (2011). Parvoviral enteritis in a dog: case report and review of the literature. Continental Journal of Veterinary Science, 5, 6-10.

[6] Haligur, M., Ozmen, O., Sezer, K., Sahinduran, S. (2009). Clinical, Pathological and Immunohistochemical Finding in Diarrheic Dogs and Evaluation of Canine Parvoviral and Coronaviral Enteritis. Journal Veterinary Advances, 8, 720-725.

[7] Pollock, R. V. H., Coyne, M. J. (1993). Canine Parvovirus. Veterinary Clinics of North America: Small Animal Practice, 23 (3), 555-568. doi: http://doi.org/10.1016/s0195-5616(93)50305-4

[8] Gombač, M., Švara, T., Tadić, M., Pogačnik, M. (2008). Retrospective study of canine parvovirosis in Slovenia. Slovenian Veterinary Research, 45 (2), 73-78.

[9] Borisevich, B. V., Lisova, V. V., Shatskaya, A. (2016). Morphological features of the myocardial form of parvovirus infection in dogs. Scientific Bulletin of LNUVMBT named after S. Z. Gzzhytsky, 18 (2), 16-19.

[10] Byun, J.-W., Yoon, S.-S., Lim, S.-K., Lee, O.-S., Jung, B. Y. (2011). Hepatic Yersiniosis Caused byYersinia Enterocolitica4:O3 in an Adult Dog. Journal of Veterinary Diagnostic Investigation, 23 (2), 376-378. doi: http://doi.org/10.1177/104063871102300233

[11] Mylonakis, M., Kalli, I., Rallis, T. (2016). Canine parvoviral enteritis: an update on the clinical diagnosis, treatment, and prevention. Veterinary Medicine: Research and Reports, 7, 91-100. doi: http://doi.org/10.2147/vmrr.s80971

[12] Kelly, W. R. (1993). Inflammation of the liver and biliary tract. Pathology of Domestic Animals. San Diego: Academic Press, 359-381.

[13] Headley, S. A., Alfieri, A. A., Fritzen, J. T. T., Garcia, J. L., Weissenböck, H., da Silva, A. P. et. al. (2013). Concomitant canine distemper, infectious canine hepatitis, canine parvoviral enteritis, canine infectious tracheobronchitis, and toxoplasmosis in a puppy. Journal of Veterinary Diagnostic Investigation, 25 (1), 129-135. doi: http://doi.org/10.1177/1040638712471344

[14] Skrypnik, V. G. (1999). Intestinal yersiniosis of animals: Library of veterinary medicine. Vol. 4. Kyiv, 48.

[15] Urbanovich, P. P., Potoc'kii, M. K., Gevkan, I. I., Zon, G. A. et. al. (2008). Pathological anatomy of animals. Kyiv: Vetinform, 896.

[16] Hayashidani, H., Kaneko, K., Sakurai, K., Ogawa, M. (1995). Experimental infection with Yersinia enterocolitica serovar 0:8 in Beagle dogs. Veterinary Microbiology, 47 (1-2), 71-77. doi: http://doi.org/10.1016/0378-1135(95)00052-c

[17] Lamm, C. G., Rezabek, G. B. (2008). Parvovirus Infection in Domestic Companion Animals. Veterinary Clinics of North America: Small Animal Practice, 38 (4), 837-850. doi: http://doi.org/10.1016/j.cvsm.2008.03.008

[18] Ivanovska, L. B. (2007). Epizootological monitoring and development of serological diagnosis of animal yersiniosis. Kharkiv, 240.

[19] Zon, G. A., Ivanovskaya, L. B. (2018). Animal autopsy handbook. Sumy: LLC «Mriya», 336.

[20] Owston, M. A., Wu, C. C., Ramos-Vara, J. A. (2006). Hepatic Yersiniosis in a Cougar (Felis Concolor). Journal of Veterinary Diagnostic Investigation, 18 (5), 511-513. doi: http://doi.org/10.1177/104063870601800520

How to cite: Zon, H., Ivanovska, L., Zon, I., Tergunzwe, T. M. (2021). Comparison of pathological changes in the study of dogs affected by parvoviral enteritis and intestinal yersiniosis. EUREKA: Health Sciences, 2, 102-110. doi: http://doi.org/10.21303/ 2504-5679.2021.001690 